\title{
Leader neurons drive spontaneous and evoked activation patterns in cortical networks
}

\author{
Valentina Pasquale ${ }^{1 *}$, Sergio Martinoia ${ }^{2,1}$, Michela Chiappalone ${ }^{1}$ \\ From Twenty Second Annual Computational Neuroscience Meeting: CNS*2013 \\ Paris, France. 13-18 July 2013
}

Recent studies about the generation and propagation of coordinated activity in cultured neuronal networks reported the existence of privileged neurons that consistently fire earlier than others at the onset of synchronized bursting events (or network bursts, NB), which have been termed major burst leaders (MBL) [1]. At the same time, by stimulating the network from different channels one can obtain very different responses, not only in size and delay but also in the activation order of the responding neurons [2].

We electrically stimulated rat cortical networks cultured on micro-electrode arrays from different locations, either MBL or non-MBL. We evaluated the intensity and the delay of responses from either MBL or non-MBL and we

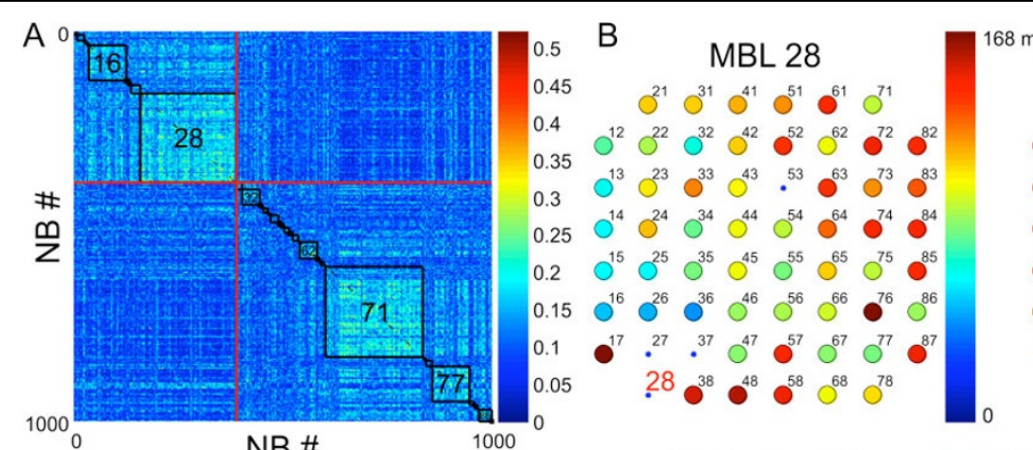

C

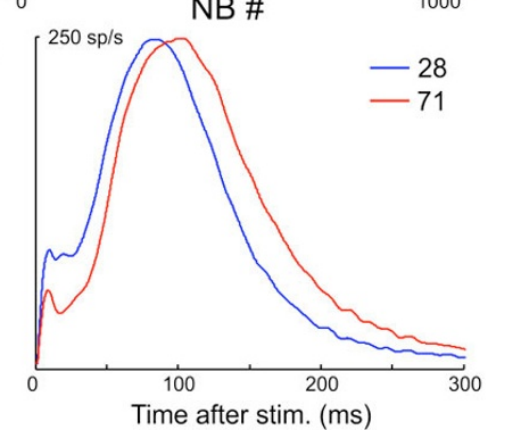

Stim. elec. 28

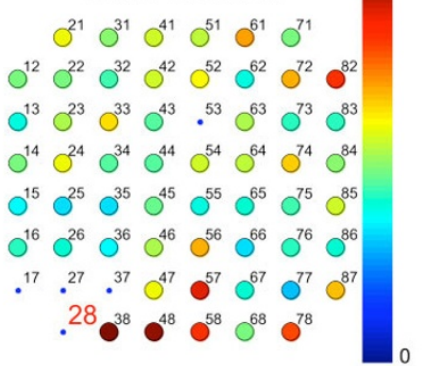

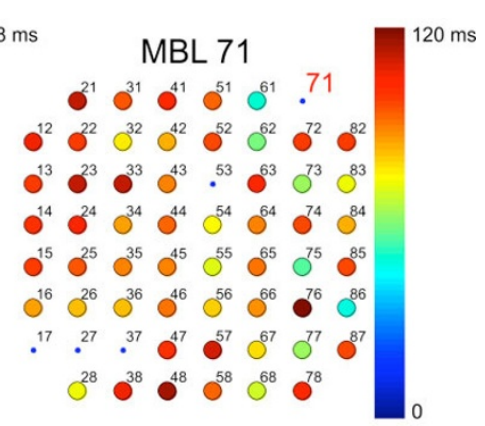

Stim. elec. 71 $\mathrm{O}^{21} \mathrm{O}^{31} \mathrm{O}^{41} \mathrm{O}^{51} \mathrm{O}^{61} \cdot \cdot^{71}$ $\rho^{12} \rho^{22} \rho^{32} \rho^{42} \rho^{52} \rho^{62} \rho^{72} \rho^{82}$ $\rho^{13} \rho^{23} \rho^{33} \rho^{43} \cdot \cdot^{53} \rho^{63} \rho^{73} \rho^{83}$ $0^{14}{O^{24}}^{34} \rho^{34} \rho^{54} \rho^{64} \rho^{74} \rho^{84}$

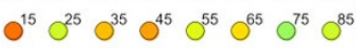
$0^{16} \rho^{26} \rho^{36} \rho^{46} \rho^{56} \rho^{66} \rho^{76} \rho^{86}$ .$\cdot^{17} \cdot \cdot^{27} \cdot \cdot^{37} e^{47} 0^{57} 0^{67} 0^{77} 8^{87}$ $0^{28} \bullet^{38} \bullet^{48} \rho^{58} \rho^{68} \rho^{78}$

Figure 1 A. Similarity matrix of activation order for 1000 spontaneous NB, ranked according to the MBL (indicated by numbers). TwO distinct propagation patterns are visible. B. Color maps of average delays of followers with respect to either MBL spontaneous activation (upper panels) or external stimulation (lower panels). Spontaneous NB starting from 28 propagate rightward, whereas NB from $71 \mathrm{go}$ leftward. NB evoked by stimulation of the same channels follow a similar pattern, although on a different timescale. C. Network average PSTH (for 28 and 71 ).

\footnotetext{
* Correspondence: valentina.pasquale@iit.it

'Department of Neuroscience and Brain Technologies - NTECH, Istituto Italiano di Tecnologia, Via Morego 30, Genova 16163, Italy
} 
also compared the spontaneous and the evoked activation patterns.

By comparing the responses obtained by stimulating either MBL or non-MBL, we found that the stimulation from MBL induces on average earlier responses. By comparing the responses of MBL and non-MBL, we found that MBL respond better and more rapidly to the stimulation coming from any other site in the network. Some networks showed different spontaneous propagation patterns within synchronized bursts depending on the identity of the corresponding MBL (Figure 1). In these cases the evoked propagation patterns correlate with the spontaneous ones and also depend on the spatial location of the stimulating site with respect to MBL.

To summarize, we demonstrated that MBLs do not only drive the propagation of coordinated spontaneous activations, but also play a special role in coordinating and driving the evoked bursts of activity.

\section{Acknowledgements}

The research leading to these results has received funding from the European Union's Seventh Framework Program (ICT-FET FP7/2007-2013, FET

Young Explorers scheme) under grant agreement $n^{\circ} 284772$ BRAINBOW.

\section{Author details}

'Department of Neuroscience and Brain Technologies - NTECH, Istituto Italiano di Tecnologia, Via Morego 30, Genova 16163, Italy. ${ }^{2}$ Department of Informatics, Bioengineering, Robotics, System Engineering (DIBRIS), University of Genova, Genova 16145, Italy.

Published: 8 July 2013

\section{References}

1. Ham Ml, Bettencourt LM, McDaniel FD, Gross GW: Spontaneous coordinated activity in cultured networks: analysis of multiple ignition sites, primary circuits, and burst phase delay distributions. J Comput Neurosci 2008, 24(3):346-357.

2. Shahaf G, Eytan D, Gal A, Kermany E, Lyakhov V, Zrenner C, Marom S: Order-based representation in random networks of cortical neurons. PLOS Comput Biol 2008, 4(11):e1000228.

\section{doi:10.1186/1471-2202-14-S1-P64}

Cite this article as: Pasquale et al:: Leader neurons drive spontaneous and evoked activation patterns in cortical networks. BMC Neuroscience 2013 14(Suppl 1):P64.

\section{Submit your next manuscript to BioMed Central} and take full advantage of:

- Convenient online submission

- Thorough peer review

- No space constraints or color figure charges

- Immediate publication on acceptance

- Inclusion in PubMed, CAS, Scopus and Google Scholar

- Research which is freely available for redistribution

Submit your manuscript at www.biomedcentral.com/submit 